\title{
Mediastinal Rhabdomyoma
}

National Cancer Institute

\section{Source}

National Cancer Institute. Mediastinal Rhabdomyoma. NCI Thesaurus. Code C6600.

A rare benign skeletal muscle neoplasm arising from the mediastinum. 